\title{
DEVELOPING FOR AND DEPLOYING WEBWORK ACROSS DISCIPLINES IN SECOND-YEAR ENGINEERING
}

\author{
Agnes G. d'Entremont ${ }^{1}$, Negar M. Harandi ${ }^{2}$, and Jonathan Verrett ${ }^{3}$ \\ Departments of ${ }^{1}$ Mechanical Engineering, ${ }^{2}$ Electrical and Computer Engineering, and ${ }^{3}$ Chemical and Biological \\ Engineering, University of British Columbia \\ agnes.dentremont@mech.ubc.ca, negarm@ece.ubc.ca, jonathan.verrett@ubc.ca
}

\begin{abstract}
Online homework systems provide immediate feedback to students, enhancing student learning. However, paid online homework from textbook publishers or other sources systems can be costly and also raise concerns about student data privacy. WeBWorK is an open-source online homework system that can be setup on local servers, is free to students and has been in use since its development in the mid-1990s. Previous to this work around 200 engineering problems were openly shared on the WeBWorK platform, limiting opportunity for adoption. In order to address this, we have developed, deployed, and evaluated nearly 1000 new engineering problems across a wide range of engineering topics at the second-year level.

Student perceptions of WeBWorK have been evaluated using surveys at the start and end of courses where it is deployed. These surveys indicate that students generally prefer the WeBWorK system to other online homework systems they have used. Surveys also indicate that students were generally motivated to both attempt and complete all assigned problems that contributed to their grade, and believed WeBWorK enhanced their learning. The creation of error-free WeBWorK questions was difficult, however the hope is that the ability to re-use and share these questions ensures they provide a higher value over the long term than paper-based homework problems.
\end{abstract}

Keywords: Online homework, open educational resources, WeBWorK, homework problem development, dynamics, mechanics of materials, fluid mechanics, electric circuit analysis, signals and systems, electromagnetism.

\section{INTRODUCTION}

Effective practice in solving engineering problems requires timely feedback, allowing students to assess and improve understanding. Large classes of over 100 students make getting timely feedback to students challenging when using paper-based assignments. Furthermore, the hours spent marking assignments could be otherwise used on instructor-student interaction. Several commercial online homework systems exist for engineering, but are typically costly for students.

Currently, there is no free, online homework source for most engineering subjects. Our project aimed to create free resources to allow fast feedback in engineering with no marking or student cost, using the WeBWorK online homework platform. The WeBWorK Open Problem Library (OPL), a WeBWorK-specific problem bank widely used in mathematics, contains 33,000+ math problems, but very few engineering problems (around 200 problems previous to this work) [1]. Students at our institution have shown preferences for WeBWorK over learning management system-based options [2]. Students surveyed in a non-WeBWorK ECE course reported that they have used the system before (91\%), rated previous WeBWorK experience positively $(88 \%)$, and valued its immediate feedback (86\%) and lack of student cost (78\%) [3].

\section{PROBLEM DEVELOPMENT}

\subsection{Goals and Target Subjects}

Our goal was to create sufficient problems to run homework for an entire course in 7 engineering subjects at the second-year level: dynamics (including basic vibrations), mechanics of materials, fluid mechanics, electric circuit analysis, signals and systems, and electromagnetism. Most of these subjects are taught in multiple disciplines, and we originally engaged instructors in mechanical, chemical and biological, electrical and computer, civil, and biomedical engineering in this project.

We assessed what material existed in the OPL, including the Contrib folder [4]. Contrib is where problem authors from various institutions can upload problems they have tested in at least one course offering. While problems in the OPL are available to instructors through the WeBWorK Library Browser interface (drop-down menus based on problem topic), problems in Contrib are not searchable in the OPL. The primary reasons for engineering problems remaining in Contrib and not moving into the OPL were a lack of engineering taxonomies to categorize problems and a lack of engineering subject matter expertise among the WeBWorK administrators (who are primarily mathematics instructors).

We also evaluated internal problems developed and used at UBC but not yet contributed to the OPL. Finally, we asked instructors whether the same subject in different departments would need discipline-specific questions. 
That is, would fluid mechanics in chemical engineering need some questions that would not be applicable to mechanical engineering, and vice versa.

Ultimately, we estimated that a course would need about 80-100 problems to run weekly homework sets with a small amount of instructor choice, and some proportion of those questions (roughly 20\%) might be disciplinespecific in some subjects. Ultimately, we planned to create 700 new problems to complement existing material and provide sufficient coverage for the 13 partner courses that agreed to adopt WeBWorK.

\subsection{Problem Development Process}

Our problem development involved faculty in five departments and employed more than a dozen students.

We hired engineering Graduate and Undergraduate Academic Assistants (GAAs and UAAs) with expertise in the subject areas to create questions. They began by consulting with individual faculty members and looking at the existing problems, if any.

The problem developers then created problems in a template that we designed to provide the coder with all the needed information about the problem (Figure 1). Some features include ranges for student variables, solution formulae, answer tolerances (see 2.3) and a set of variables that provide a numerical check of the formulae.

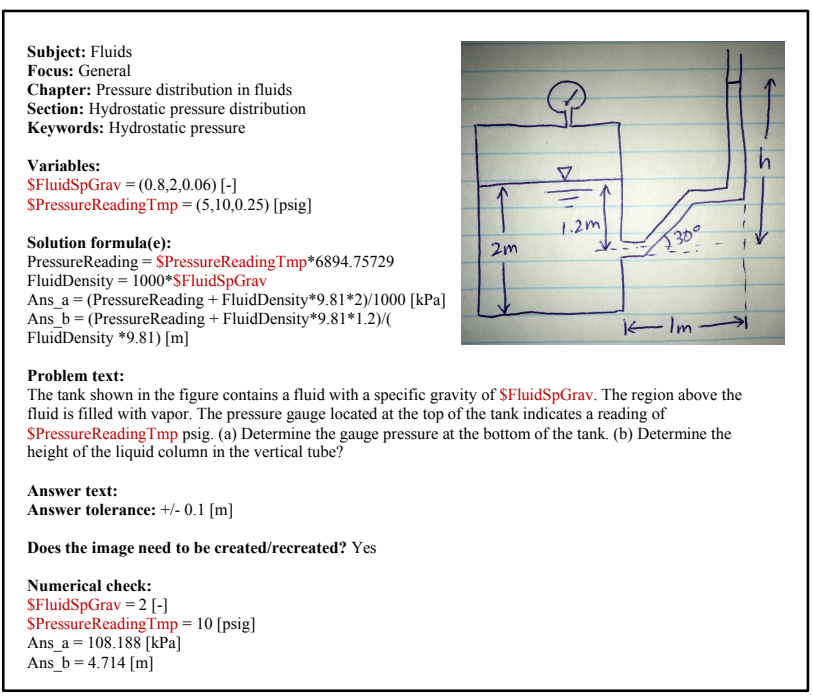

Figure 1: Sample completed template for one problem.

One co-op student was hired as coder for the project. This student did all the coding and the majority of the graphics creation, as well as providing part-time support during course offerings. About $50 \%$ of the problems required creation of images/diagrams/plots and we applied Creative Commons licensing to all graphics (Figure 2).

Following coding, problems were checked by the problem authors, and any required corrections were made. Overall, each new problem took between 2 and 3 total hours of work, depending on images, etc.

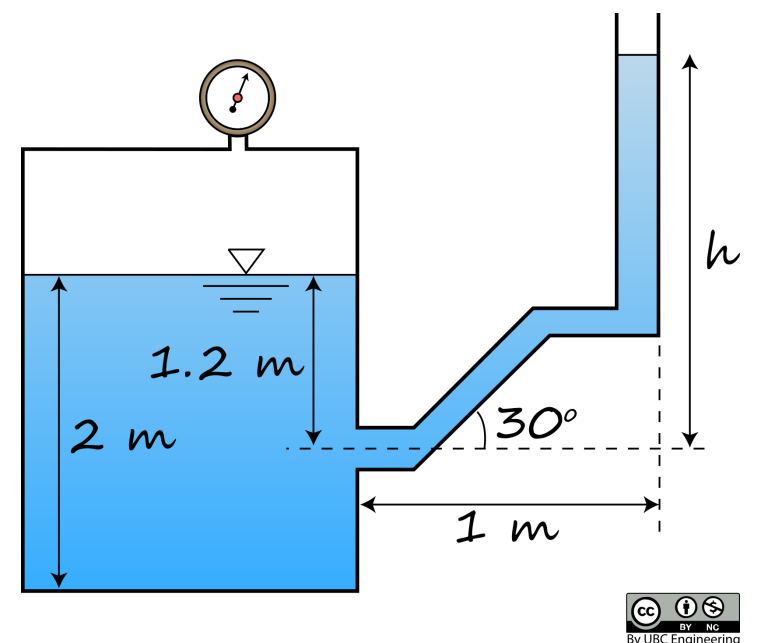

Figure 2: Sample completed problem image.

Problems were deployed in courses in both terms of the 2018-2019 academic year (Figure 3). Support was provided to courses to deal with errors and ambiguities, tolerance issues, and system management.

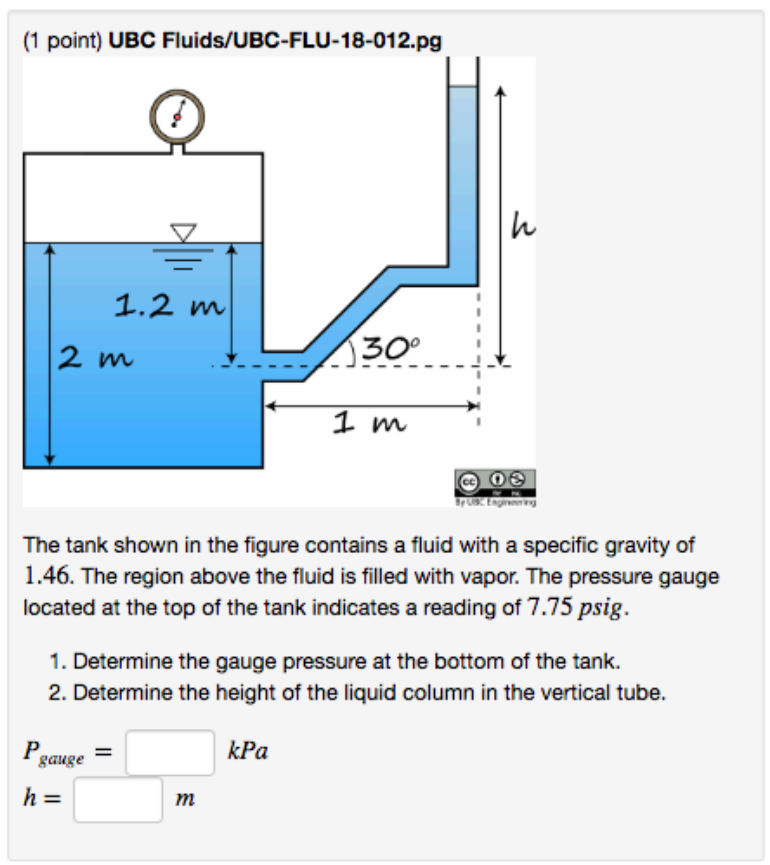

Figure 3: Sample completed problem.

We submitted any fully tested problems (i.e. tested in a course; see 2.3.1) for which we had a taxonomy to the OPL Contrib folder. With the exception of 25 problems used with permission from a colleague's published book, all problems have Creative Commons licenses. Finally, we evaluated the project.

\subsection{Challenges}

One challenge was limited instructor time to provide guidance to the student problem developers. Instructor 
input was critical to creating problems relevant to the course in terms of content and level of difficulty. As a result, some problems were delayed such that they could not be deployed to students during this school year.

Some of the problem developers chose to focus on very challenging problems, and we found that the there is a need to add further introductory problems in order to create a learning progression within the homework or practice sets.

We found that the Signals and Systems problems were particularly difficult to code, as they involved dynamic graphs (unique graphs plotted for each student). As a result, we reduced the target number of problems to be created.

Calculated-answer problems with unique variables for each student require coders to set tolerances of acceptable numerical values. For example, intermediate-step rounding could lead a student to submit 42.15 when the exact answer would be 42.1923. Tolerances are expressed either as percentages or absolute values. Setting tolerances to exclude incorrect methods but allow rounding errors was difficult, particularly in some subjects (e.g. mechanics of materials: physical component length change is often a small percentage).

2.3.1. Testing. We found in the first term of deployment that, while the problem authors (UAAs and GAAs) did check their own work, an unacceptably large number of errors still existed in the problems. This is likely partly due to the difficulty of seeing one's own mistakes or omissions.

As a result, we obtained additional resources to improve testing. This included our co-op student coder working through the fluid mechanics problems, and hiring upper year undergraduate students to work through the electromagnetism and circuits problems, prior to deployment in courses in the second term. Instructors performed testing of the dynamics and some of the mechanics of materials problems prior to deployment. Once checked/corrected, the problems will not require ongoing work. This additional testing need was unanticipated, and we recommend such testing should be considered when planning for online homework development.

The final level of testing was within the courses. As much as we attempted to make the problems error-free, students ultimately found issues. Our student coder was able to respond quickly when issues were reported in order to push out corrections.

\subsection{Synergies and Collaborations}

We were able to translate fluid mechanics problems created by colleagues at Queen's University from the Desire2Learn learning management system into WeBWorK for use at UBC and sharing in the Open Problem Library (OPL). Similarly, we converted statics problems created by a colleague at Whatcom Community College from the IMATHAS system into WeBWorK for use at UBC and posting to the OPL.

\section{PROBLEMS CREATED, CONVERTED, AND ADDED TO THE OPL}

\subsection{Problems Developed/Coded}

We currently have 1372 engineering problems either added to the OPL or being tested and prepared for submission (Figure 4). This is a combination of newly coded problems ( 950) and hundreds of existing internal problems that were modified to allow posting. We completed the translation of 190 additional fluid mechanics problems from the Desire2Learn learning management system and 41 statics problems from the IMATHAS system into WeBWorK. The problems have been uploaded to the Contrib folder of the OPL [4]. The translation scripts for Desire2Learn are available openly to allow others to perform similar conversions [5].

The distribution of problems, including those from other authors currently in the OPL and those converted by UBC from collaborators, is presented in Figure 4.

\subsection{OPL Subject Areas}

Related to this project, we have gotten five new engineering subject areas added to the overall OPL taxonomy, and successfully submitted/administered problems in those areas. These areas are: dynamics, fluid dynamics, material and energy balances, thermodynamics and vibrations. We also split and expanded a previously combined taxonomy for statics and mechanics of materials [6]. We have increased the OPL engineering content to 868 problems in 8 engineering subjects currently, up from $\sim 200$ problems in three subjects. This involved performing the administrative tasks required, including creating taxonomies. We were also able to include problems into the OPL that had been contributed by other schools, but were stuck in Contrib without taxonomical support or administrator subject matter expertise.

\section{PROJECT EVALUATION}

Our project evaluation had three main thrusts: (a) determining student priorities and preferences for online homework broadly, (b) determining the impact of the new problems on students and instructors, and (c) evaluating the problem development and deployment process.

\subsection{Student Pre-surveys}

The focus of the pre-surveys was to determine current attitudes toward WeBWorK (if used in the past) and other homework systems (if used in the past) (Table 1), as well as student priorities when it comes to online homework systems (Table 2). The same survey was deployed in term $1(\mathrm{n}=180)$, and in select term 2 courses where the majority would not have been invited in term $1(\mathrm{n}=169)$. A MannWhitney $U$ test was used to compare student ratings of WeBWorK and other systems - for many items, WeBWorK was rated significantly better (Table 1). 


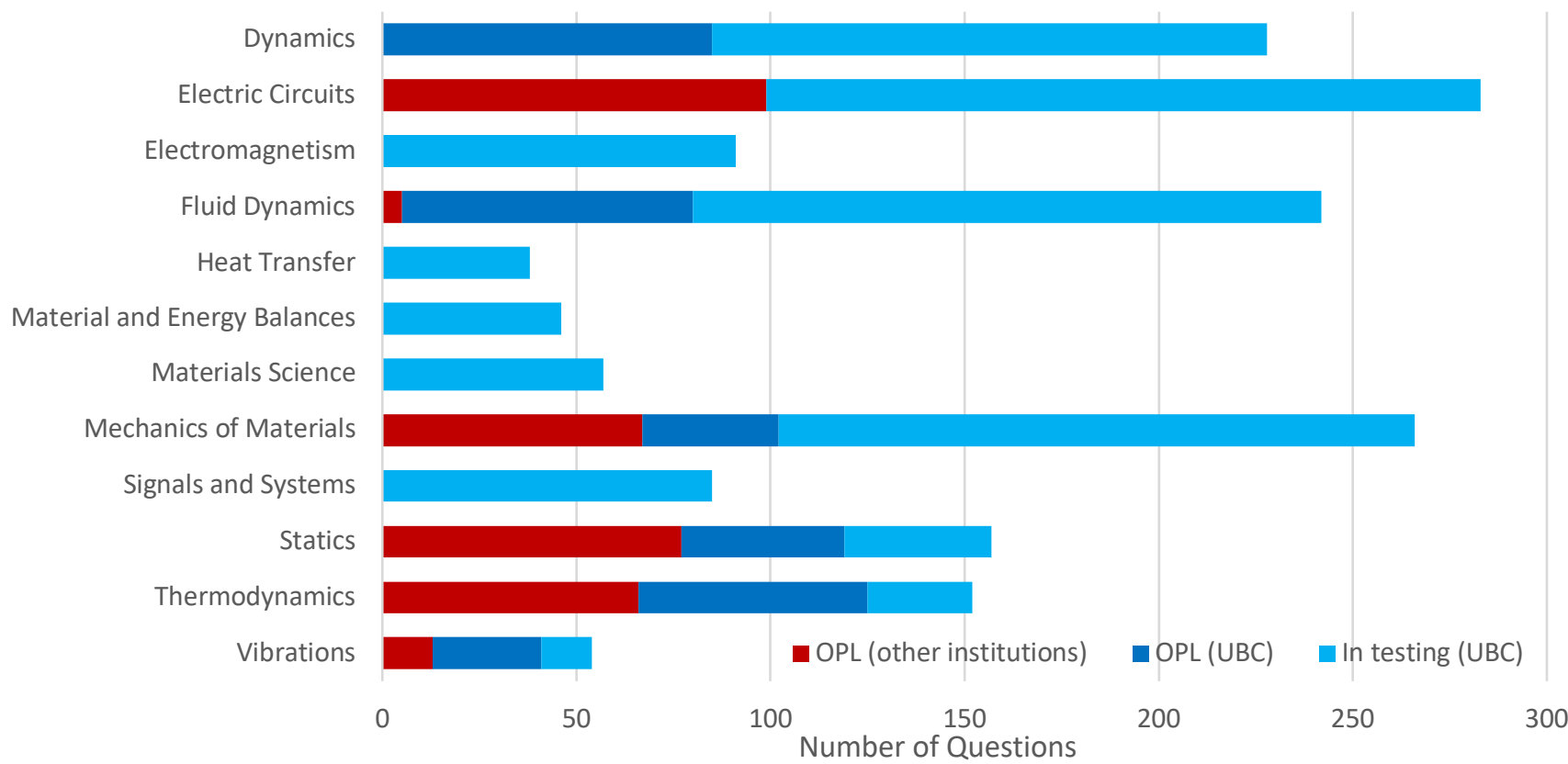

Figure 4: Summary of engineering questions in the OPL and in testing at UBC in preparation for submission.

Table 1: Combined term 1 and term 2 results for past experience with online homework systems (5=strongly agree, $1=$ strongly disagree). ${ }^{*}$ Significantly higher (Bonferroni-corrected: $p<0.0063$ )

\begin{tabular}{|l|c|c|}
\hline $\begin{array}{l}\text { Rate your experience with } \\
\text { online homework systems in } \\
\text { the past, considering the } \\
\text { most recent time. }\end{array}$ & $\begin{array}{c}\text { WeBWorK } \\
\text { (n= 320) }\end{array}$ & $\begin{array}{c}\text { Other } \\
\text { Systems } \\
\text { (n= 295) }\end{array}$ \\
\hline The system was easy to use & $\mathbf{4 . 3 *}$ & 3.7 \\
\hline The site was easy to navigate & $\mathbf{4 . 2 *}$ & 3.7 \\
\hline $\begin{array}{l}\text { The question presentation was } \\
\text { clear }\end{array}$ & 4.0 & 3.9 \\
\hline $\begin{array}{l}\text { The required answer formats } \\
\text { were straightforward }\end{array}$ & 3.5 & 3.7 \\
\hline $\begin{array}{l}\text { The feedback was clear and } \\
\text { easy to access }\end{array}$ & 3.3 & $\mathbf{3 . 8 *}$ \\
\hline $\begin{array}{l}\text { The system enhanced my } \\
\text { learning }\end{array}$ & $\mathbf{4 . 0 *}$ & 3.7 \\
\hline $\begin{array}{l}\text { The system motivated me to } \\
\text { persist in finding the correct } \\
\text { answer }\end{array}$ & $\mathbf{3 . 8 *}$ & 3.6 \\
\hline $\begin{array}{l}\text { I would like to use the system } \\
\text { in the future }\end{array}$ & $\mathbf{4 . 0}^{*}$ & 3.2 \\
\hline
\end{tabular}

The open comments on past homework systems mentioned appreciating WeBWorK's simple interface, clear feedback, and unlimited attempts. Challenges mentioned with WeBWorK include errors in problems, lack of detailed feedback (including on intermediate steps), the required syntax, inconsistency in question style (different authors), automatic sign-outs, and lack of good mobile support. A majority of comments on other homework systems mentioned their high cost, and many indicated that the quality increase over WeBWorK was not worth the price, although some appreciated hints/guidance, the adaptive presentation of additional problems, and the better user interface of these systems. They also mentioned disliking using multiple systems for different courses.

Table 2: Combined term 1 and term 2 results for student priorities ( $5=$ extremely important, $1=$ not at all important).

\begin{tabular}{|l|c|}
\hline $\begin{array}{l}\text { How important are the following factors to you } \\
\text { when it comes to online homework systems? }\end{array}$ & $\mathbf{n}=\mathbf{3 3 1}$ \\
\hline The system is easy to use & 4.5 \\
\hline The system is low cost or free & 4.7 \\
\hline The system is open source & 3.4 \\
\hline $\begin{array}{l}\text { The same system is used by many (or all) of my } \\
\text { courses }\end{array}$ & 3.7 \\
\hline $\begin{array}{l}\text { The system motivates me to correctly solve } \\
\text { problems/complete homework }\end{array}$ & 4.3 \\
\hline $\begin{array}{l}\text { The system is integrated with the learning } \\
\text { management system }\end{array}$ & 3.7 \\
\hline The system is integrated with my textbook & 2.9 \\
\hline $\begin{array}{l}\text { The system suggests targeted additional practice } \\
\text { problems based on my results }\end{array}$ & 3.5 \\
\hline $\begin{array}{l}\text { The system provides hints within problems based } \\
\text { on my results }\end{array}$ & 3.9 \\
\hline
\end{tabular}


Open comments, when asked about other homework system priorities, mentioned better integration with (or complete avoidance of) learning management systems, good usability, completion badges (stars) as motivation, hints as motivation, suggesting targeted additional practice problems, ability to mark questions for review later, offline use/PDF downloads, links to resources (textbook) and answer previews for syntax checks.

\subsection{Student Post-surveys}

In the post-surveys, the focus was on potential issues with the problems (Table 3), how motivated students were to complete work on WeBWorK and student beliefs about impact on learning (Table 4), and how often students interacted with others (instructors, TAs, peers) about WeBWorK (Table 5). When asked if engineering instructors should continue to use WeBWorK in similar courses, students agreed (7.7/10, median 8$)$.

Table 3: Combined term 1 and term 2 results about the WeBWork problems used this term ( $5=$ strongly agree, $1=$ strongly disagree).

\begin{tabular}{|l|c|}
\hline $\begin{array}{l}\text { Please rate how you agree or disagree with the } \\
\text { following statements about issues with the } \\
\text { problems you used this term. }\end{array}$ & \\
\hline $\begin{array}{l}\text { The problems had few errors (missing values, } \\
\text { incorrect answers, etc.) }\end{array}$ & 2.8 \\
\hline The problems had few issues with answer tolerances & 3.1 \\
\hline $\begin{array}{l}\text { Problem text was almost always clear and } \\
\text { understandable }\end{array}$ & 3.6 \\
\hline $\begin{array}{l}\text { Errors or other issues with the problems were } \\
\text { corrected quickly }\end{array}$ & 3.2 \\
\hline $\begin{array}{l}\text { The problems were at the same level of difficulty as } \\
\text { tests/exams }\end{array}$ & 2.9 \\
\hline $\begin{array}{l}\text { The problems were more challenging than } \\
\text { tests/exams }\end{array}$ & 3.2 \\
\hline Images were almost always clear and understandable & 4.1 \\
\hline $\begin{array}{l}\text { The instructor(s) and/or TA(s) knew how to use the } \\
\text { system }\end{array}$ & 3.6 \\
\hline
\end{tabular}

Student comments reflected that errors and issues with tolerances were common and frustrating. These were generally fixed on the fly while the homework was open for submission. Students did comment positively about the responsiveness in correcting issues. Some comments mentioned instructors and TAs not being familiar with the system, and the problem level of difficulty not matching the class examples or tests. As in the pre-survey, there were a few comments about the negatives of getting "right/wrong" feedback only, and wanting hints and staged questions with multiple answer boxes. Students again mentioned lack of cost and multiple attempts as positives.

When commenting on motivation and learning, students mentioned WeBWorK helping them keep on top of classwork, allowing troubleshooting of work, prompting studying to understand material in the problems, and being motivated by the badges (stars) showing completion. Some students said problems were harder than exams, and some said they were easier, highlighting differing pedagogical uses of WeBWorK by instructors. Students mentioned wasted time when there were errors in problems, or that they could not find the errors in their own work.

Table 4: Combined term 1 and term 2 results about motivation, leanring and WeBWorK ( $5=$ strongly agree, $1=$ strongly disagree).

\begin{tabular}{|l|c|}
\hline $\begin{array}{l}\text { How did WeBWorK impact your studies this term } \\
\text { in your engineering courses? }\end{array}$ & \\
\hline $\begin{array}{l}\text { I was motivated to attempt to solve all problems that } \\
\text { counted for marks }\end{array}$ & 4.3 \\
\hline $\begin{array}{l}\text { I was motivated to attempt to solve all problems that } \\
\text { did not count for marks }\end{array}$ & 2.9 \\
\hline $\begin{array}{l}\text { I was motivated to successfully complete all } \\
\text { problems that counted for marks }\end{array}$ & 4.2 \\
\hline $\begin{array}{l}\text { I was motivated to successfully complete all } \\
\text { problems that did not count for marks }\end{array}$ & 2.7 \\
\hline I was motivated to correct my errors in understanding & 3.8 \\
\hline $\begin{array}{l}\text { I expect using WeBWorK will help me to be well } \\
\text { prepared for the final exam in this course }\end{array}$ & 3.8 \\
\hline I enjoyed using WeBWorK & 3.4 \\
\hline I believe WeBWorK enhanced my learning & 4.0 \\
\hline
\end{tabular}

Table 5: Combined term 1 and term 2 results about interactions based on WeBWork problems ( 5 = weekly or more often, 1 = never).

\begin{tabular}{|l|c|}
\hline $\begin{array}{l}\text { How frequently did you do the following this past } \\
\text { term in your engineering courses? }\end{array}$ & \\
\hline Discussed WeBWorK problems with classmates & 4.3 \\
\hline Visited a TA to ask about a WeBWorK problem & 2.9 \\
\hline $\begin{array}{l}\text { Emailed/messaged a TA to ask about a WeBWorK } \\
\text { problem }\end{array}$ & 4.2 \\
\hline $\begin{array}{l}\text { Emailed/messaged an instructor to ask about a } \\
\text { WeBWorK problem }\end{array}$ & 2.7 \\
\hline $\begin{array}{l}\text { Visited an instructor to ask about a WeBWorK } \\
\text { problem }\end{array}$ & 3.8 \\
\hline $\begin{array}{l}\text { Posted about a WeBWorK problem on a class } \\
\text { discussion board }\end{array}$ & 3.8 \\
\hline Other & 3.4 \\
\hline
\end{tabular}

Under the "other" open text box about activities related to the homework system, students commented that they also used class discussion boards, asked instructors to walk through problems in class, and asked instructors to better review the level of the problems before assigning them. Some received information from peers on errors, and looked for tutorials and other help online. A few students also mentioned copying work and entering random values.

The final open comment box attracted a large number of comments, mainly similar to the above. Many comments simultaneously acknowledged the learning benefits and the errors. Students generally identified that they liked the platform with its instant feedback and lack of cost, but had concerns with matching homework content and level to coursework and testing, and a desire for targeted hints. Some explicitly mentioned it was worth developing these 
new engineering WeBWorK problems, and that they would be very useful once fully tested and error-free. A few students said they would prefer paper-based homework and learning through textbook problems.

\subsection{Instructor Feedback}

Informal feedback from instructors has indicated that errors in code and tight tolerances caused challenges. In some subjects, questions as created by problem developers were uniformly difficult/high-level and more foundational questions are needed. Instructors indicated that it was very useful to have on-call support for the new problems, allowing quick corrections. Overall, WeBWorK freed time from marking and/or provided formative feedback that wasn't available to students before (in courses where there were no assignments/marking hours previously). Instructors generally indicated that they would keep using the system.

\section{DISCUSSION AND CONCLUSIONS}

Overall, our evaluation results have confirmed the usefulness of this project. Students, confronted with increasing educational costs, value quality educational resources provided at low or no cost. Additionally, informal results indicate that instructors value being able to provide formative feedback to students, again in a context of limited support resources for marking. Instructors can also customize problems for specific courses/topics, and share problems globally.

Our evaluations results are consistent with work in the literature which shows that students who prefer online homework specify factors such as instant feedback [7]-[9], and multiple attempts [7] in their preference, and indicate that they feel the online homework system improved their learning [10].

Based on past experience with homework systems, the students surveyed preferred WeBWorK in several aspects. In addition, students during the term were generally motivated to both attempt and complete all assigned problems that contributed to their grade, and believed WeBWorK enhanced their learning.

We found that creating new, error-free problems in WeBWorK was time- and resource-intensive. Students noted that there were errors and tolerance issues in the problems they used, which were fixed during the courses.

The ability to amortize the cost of problem development over years of use, potentially in many institutions, means that the overall cost per course offering is low. If the cost of a question (2.5 hours) is $\sim \$ 60$ worth of student work, and about 80 questions are used per course, the total cost to develop WeBWorK problems for an entire course is about $\$ 4800$. If students would otherwise pay $\$ 70$ per course for access to commercial online homework, the WeBWorK development cost is equal to what a 70-person class pays once. So, while upfront costs are high, the longterm savings for students are substantial.

For instructors planning to develop WeBWorK problems, rigorous testing prior to deployment is critical. Problems took longer to develop than expected, which was partly due to instructor availability, and partly due to additional testing that we implemented. For instructors who wish to use our problems, fully tested problems will be posted to the OPL following finalization during summer 2019. We are also happy to share problems still in testing (with the caveat that errors may still be present).

In conclusion, we developed, deployed, and evaluated nearly 1000 new WeBWorK problems for second-year engineering. We would encourage instructors using or thinking about using online homework in engineering to consider WeBWorK.

\section{Acknowledgements}

This work was completed on the traditional, ancestral, and unceded territory of the Musqueam people.

We gratefully acknowledge the financial support for this project provided by UBC Vancouver students via the Teaching and Learning Enhancement Fund. We also gratefully acknowledge additional funding support from BCcampus and the UBC Applied Science Dean's Office.

We would like to thank all the instructors, academic assistants and staff who worked to create these problems and support the project, especially our coder and graphicsmaker Gianni Co.

Finally, we appreciate the patience and support of the many UBC students who worked through the new problems during their courses in the 2018-2019 school year.

\section{References}

[1] "WeBWorK Documentation Wiki Open Problem Library." [Online]. Available: http://webwork.maa.org/wiki/National_Problem_Librar y. [Accessed: 10-Jan-2017].

[2] A. G. d'Entremont, P. J. Walls, and P. A. Cripton, "Student Feedback and Problem Development for WeBWorK in a Second-Year Mechanical Engineering Program," in Canadian Engineering Education Association Annual Meeting, 2017, p. 2-7, Paper 33.

[3] N. M. Harandi, "Unpublished results from UBC Electrical and Computer Engineering survey on WeBWorK.” 2017.

[4] "WeBWorK 'Contrib' folder, Open Problem Library." [Online]. Available:

https://github.com/openwebwork/webwork-openproblem-library/tree/master/Contrib.

[5] G. Co, "WeBWorK Scripts," 2019. [Online]. Available: https://github.com/ubc-mech2/misc-webwork-scripts.

[6] A. G. d'Entremont and J. Abello, "Creating problem taxonomies for WeBWorK in mechanical engineering," in American Society for Engineering Education Annual Meeting, 2018, p. Paper \# 21641. 
[7] J. Griggs, "Effects of a Web-based Homework Delivery and Submission System on Student Achievement and Student Attitudes in a One-semster Calculus Course," Chem. Rev., vol. 96, pp. 1533-1554, 1996.

[8] L. Wang, "Teaching differential equations using WeBWorK as an online tool," in E-Learn, 2018, pp. 1089-1092.

[9] M. K. Swanbom, D. W. Moller, K. Evans, and T.
Reeves, "Open-source, online homework for statics and mechanics of materials using WeBWorK: assessing effects on student learning," ASEE Annu. Conf., 2016.

[10] D. Nguyen, Y. Hsieh, and G. Allen, "The impact of web-based assessment and practice on students' mathematics learning attitudes," J. Comput. Math., vol. 25, pp. 251-279, 2006. 